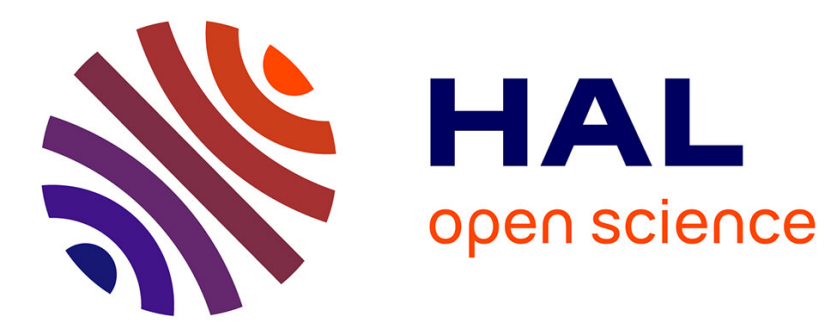

\title{
Les dépôts éocènes et leur signification morphologique en Bretagne méridionale et dans le Massif vendéen
}

\author{
Jean-Marie Bourdeau, A. Guilcher
}

\section{To cite this version:}

Jean-Marie Bourdeau, A. Guilcher. Les dépôts éocènes et leur signification morphologique en Bretagne méridionale et dans le Massif vendéen. Bulletin de l'Association de géographes français, 1938, 117, pp.136-139. 10.3406/bagf.1938.7018 . insu-01782534

\section{HAL Id: insu-01782534 \\ https://hal-insu.archives-ouvertes.fr/insu-01782534}

Submitted on 4 Mar 2021

HAL is a multi-disciplinary open access archive for the deposit and dissemination of scientific research documents, whether they are published or not. The documents may come from teaching and research institutions in France or abroad, or from public or private research centers.
L'archive ouverte pluridisciplinaire HAL, est destinée au dépôt et à la diffusion de documents scientifiques de niveau recherche, publiés ou non, émanant des établissements d'enseignement et de recherche français ou étrangers, des laboratoires publics ou privés.

\section{()ㅜ) $\Theta$}

Distributed under a Creative Commons Attribution - NoDerivatives| 4.0 International 


\section{Les dépôts éocènes et leur signification morphologique en Bretagne} méridionale et dans le Massif Vendéen. Note préliminaire Jean-Marie Bourdeau, André Guilcher

\section{Citer ce document / Cite this document :}

Bourdeau Jean-Marie, Guilcher André. Les dépôts éocènes et leur signification morphologique en Bretagne méridionale et dans le Massif Vendéen. Note préliminaire. In: Bulletin de l'Association de géographes français, $N^{\circ} 117,15 e$ année, décembre 1938. pp. 136-139;

doi : https://doi.org/10.3406/bagf.1938.7018

https://www.persee.fr/doc/bagf_0004-5322_1938_num_15_117_7018

Fichier pdf généré le 25/04/2018 
Communication écrite de MM. J.-M. Bourblice et A. Gencinen

\author{
Les dépóts éocines et leur signification morphologique \\ en Bretagne Méridionale et dans le Massif Vendéen
}

\title{
Note préliminaire
}

Des recherches et observations communes, effectuees en BasseBretagne, dans le Pays Nantais et en Vendée, nous ont anenés a considérer qu'il existait dans tout le Sud du Massif Armoricain des relations entre les dépôts éocènes et la morphologie. Ces rela-. tions ont été parfois notées pour les dépôts marins; seul M. Milon en a signalé quelques unes pour les dépots continentaux. Ces derniers se presentent presque toujours sous la forme de « grès quartzites a ciment de silice fibreuse ou de quart\% $\gg$, à grain plus ou moins fin, souvent « enrobés dans des argiles blanches et des glaises jaunatres. Ils sont fréquemment ferrugineux et leur surfice, patinée et lustrée, plus ou moins rougeâtre, témoigne d'une action xérothermique. Cerlains sont d'énormes blocs de. plusieurs centaines de kilogrammes; les autres, souvent a facetles, sont besucoup plus petits (en géneral $0 \mathrm{~m}$. 05 à $0 \mathrm{~m}$. 40). C'est l'aspect typique des grès à Sabalites andegavensis qu'on s'accorde aujourd'hui à dater de l'éocène moyen.

1) Bretagne Méridionale. - On a déjà reconnu l'éocène marin aux rochers du four, de la Banche et du Banc de Giuerande en face le Croisic, et près de Port-Iouis; l'anse de Toulven, au Sud de Quimper, qui contient des grès à Sabalites a connu aussi un episode marin. I'un de nous a également signalé en 1937 (1) des galets de silex et de calcaire pres de lont-l'Abbe. Quant a l’écène continental, il a été reconnu au Sud de la Montagne Noire, oì il couvre de vastes surfaces dans la région de PlourayI.angonnet et dans celle de Guiscriff-Coadri, ainsi que sur les rives Est et Sud-Est du Golfe du Morbihan. Nos recherches nous ont fait découvrir de nouveaux gisements aux endroits suivants : a Saint-Colombier et la Saline (Nord de Saint-(iildas de Rhuys) 'n continuite avec les autres formations du Golfe du Morbihan ; sur la crète de Grandchamp, au Nord de Vannes, a l'intérieur du Camp de Meucon et a 2 km. environ au Sud-Ouest des bâtiments du Camp; enfin au Sud de Rostrenen, près de Bonen.

Si les dépôts marins ne dépassent pas beaucoup le niveau marin actuel (entre 0 et $40 \mathrm{~m}$. environ), par contre les gre's se rencontrent a de's altitudes tres variables: de $(j$ à $30 \mathrm{~m}$. environ autour ciu (iolfe du Morbihan, de 125 a $145 \mathrm{~m}$. au Camp de Meucon, 180 mètres à Bonen, $17 j$ à $200 \mathrm{~m}$. à Plouray-I angonnet, 150 à $240 \mathrm{~m}$. i Guiscriff-Coadri.

Ces derniers en particulier, en une région de plateaux à rivieres non encaissées, montent du Sud au Nord de 150 a $200 \mathrm{~m}$. environ, mais au Sud-Ouest ils se relèvent trìs brusquement le

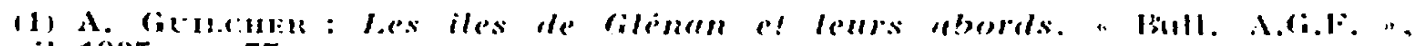
avil 19:37. p. 77. 
long d'une ligne orientée E. $135^{\circ}$, vers Coadri, sur le flanc d'une colline au sommet aplani et recouvert de nombreux grès typiques. Il semble bien que cette colline est un horst post-éocène étant donné la présence des mêmes grès aut sommet et au pied. Les hauteurs de granulite, dominant au. Sud les schistes recouverts de ces grès, se relèvent d'ailleurs dans le prolongement de la colline de Coadri, ce qui semble confirmer son caractère de horst et la préexistence des hauteurs granulitiques au-dessus des schistes.

Quant aux dépôts de Plouray, ils sont localisés en une cuvette dont aucun des bords n'est dî̀ à l'érosion différentielle ; notamment la Montagne Noire, crête appalachienne, en est séparée par un replat très net au pied de la limite structurale. On doit voir là un graben post-éocène qui aurait joué à peu près selon deux décrochements hercyniens. La Montagne Noire dominant le replat, témoin de la zone non effondrée, en est en droit de penser que ce relief appalachien existait dès l'éocène.

Les dépòts de Bonen se trouvent également en une cuvette formée des mêmes roches que les zones encadrantes. Ils affleurent près de la ferme de Kercharles, ayant dû glisser vers le Blavet par solifluction ou ravinement, mais ils sont certainement presque en place.

Enfin, à 10 km. de distance, on rencontre les grès à $145 \mathrm{~m}$. au Camp de Meucon et sur l'estran dans le Golfe du Morbihan (Conloau). On ne peut invoquer une coulée de grès dans un head, car cette coulée, d'ailleurs d'amplitude énorme, aurait dî̀ remonter la rive Sud du Golfe pour déposer les grès à l'Est d'Arzon. II doit $y$ avoir encore ici mne dislocation post-éocène, peut-être en rapport avec: le grand filon de quartz de Bretagne Méridionale.

Tout ceci, joint à l'existence d'une autre cuvette au Sud de Bonen, à Saint-Tugdual-Ploërdut, et au fait que les dépôts de Toulven se trouvent dans une dépression également non appalachienne, amène à penser à des déformations d'une surface cocéne dénivelée par des mouvements postérieurs et à l'existence de crêtes appalachiennes dominant le pays de l'éocène (1).

2) Pa!js Nantais et Vendée. - L'existence de dépôts éocènes sur la bordure du Massif Vendéen, dans le Marais Vendéen et sur la côte a été signalée depuis longtemps. L'altitude même de l'Auversien marin au contact du Bocage et du Marais (- $10 \mathrm{~m}$. dans la Baic de Bourgneuf, $+40 \mathrm{~m}$. à Arthon, à Machecoul et aux Moutiers) devait suggérer l'hypothise de dislocations postérieures à son dépôt (2). I a position et l'extension des dépôts eocènes continentaux permettent d'être pluis aflirmatif. I.es grès à Sabalites du Bois de la Chaize, à Noirmoutier, sont bien connus ;

1) Lne certe appalachienne traversant lo bassin de Saint-Tugdual pourrait avoir cette origine nnciente et s'etre trouves denivelec pin rapport atux plateaux environnants m nenge temps que lo bassin.

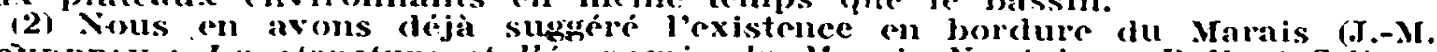

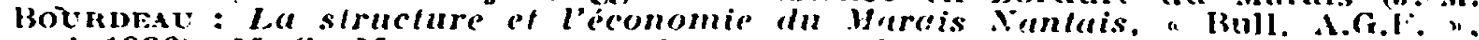

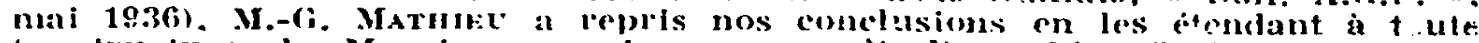
la structure du Marais, re qui nous parait discutable ic:. Natriet: I.es cifformations récentes diu sol dans le ...W. de la Vendéc. C.R. Acad. St... t 206, p. 1.128. I avril 1938). 
la carte géologique (Nantes, 117) en indique aussi quelques gisements au Sud du Lac de Grand-Lieu. En réalité, la formation est beaucoup plus étendue et nouts avons trouvé des grés (gros blocs oll fragments), en place ou presque en place dans tout l'Ouest du Mussif Vendéen. Trois gisements sont particulièrement remarquables : celui de Touvois-Legé où les grès sont en place sur le Primaire (Ie Falleron, Frérigné, la Péranche, la Morlière) ou sur le 'Turonien (Forêt de Touvois); celui de la vallée inférieure de la Vie, où nous les avons rencontrés sur le Primaire et surtout le Cénomanien dont ils se distinguent nettement, dans les environs d'Apremont, Saint-Maixent sur Vie, Commequiers et sur l'ancienne île de Notre-1)ame de Riez; enfin dans le Bassin de Chantomay et ses abords, de part et d'autre de la grande faille méridionale, les gros blocs sont abondants sur le Primaire (Mou(hamps, Saint-Martin des Noyers, les Clous) et sur le Lias ou le Jurassique (Pont-Charron près Sainte-Cécile, (hantonnay, la (aillère, Saint-Vincent-Sterlanges, Saint-Mars des Près); plús autu Nord, de petits grès en place dans l'argile à $3 \mathrm{~km}$. au Sud du lioupere, sur le primaire, offent une analogie frappante avec le gisement du Camp de Meucon. Enfin des blocs recoubrent aussi icocene marin a Sallertaine et dans la carrière du Molin, à l'Est de Challans. 'Tous ces gisements se raccordent par des témoins isolés.

Nous nous permettons de signaler comme une hypothèse séduisante linterprétation de concretions plus ou moins siliceuses surmontant de nombreuses carrières comme une formation éocène ou le résultat d'un remaniement éocène: les mêmes rognons recouvrent le calcaire jurassique a Saint-Vincent-Sterlanges; les sables cénomaniens a la Fonteny (Ouest de la Garnache) et le cal(aire auversien au Molin (1).

Ainsi les dépots continentaux eocenes du Massif Vendéen, en relation de continuité vers le Nord avec ceux de la Bretagne Méridionale (par les gisements de Touvois, Saint-Michel Chef(hef, Nantes-Doulon, Berreau-en-Misillac), occupent une aire stenclue limite vaisemblablement au Sud par une ligne Saint(iilles sur Vie-Bassin de Chantonnay, et à l'Est par les hauteur's du Haut-IBocage, sur lequel nous n'avons trouvé aucun dépot (ce qui exclut un raccordement avec les gisements de l'Anjou).

lees différences d'altitude des dépôts $(-10 \mathrm{~m}$. dans la Baie de Bourgneuf, $+110 \mathrm{~m}$. a Saint-Martin des Noyers), leur position recoupant a la fois le Primaire, le Jurassique, le Crétacé, l'Eocinc marin, leur situation de part et d'autre des grands accidents suggèrent lidée d'une vaste surface éorène dénivelée par de's dislocations postérienres : elle a notamment fossilisé les dépôts cénomaniens conserves malgré leur peu de resistance; on la retrouve sans doute, gauchic ef inclinee du Nord-Est au SudOuest, constituant les plateaux du Bas-Bocage. Des monvements post-écoines, faisant rejouler parfois des accidents hercyniens.

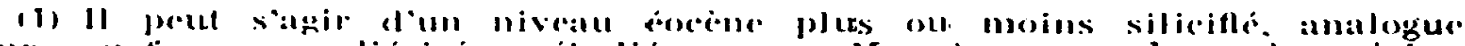

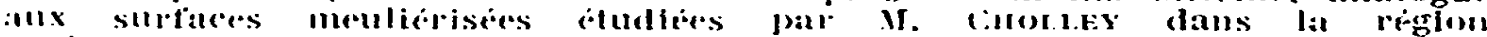

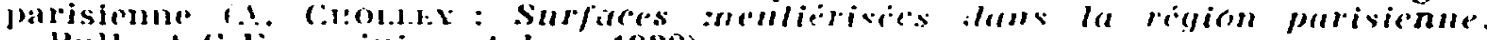
- Hull. A.(i.l. . juin-r(w)te $29 ? 8)$. 
l'ont démantelée; c'est le cas de la grande faille de Chantonnay dont la fraîcheur de formes témoigne d'un rejeu récent. Dans la région côtière ils ont dénivelé une série de blocs : certains ont basculé (Pays de Retz limité au Sud-Ouest par une dislocation N.-E.- S.-W. correspondant à la ligne de relief Machecoul-Pointe Saint-Gildas, ancienne presqu'île de Beauvoir-Challans, ille d'Yeu, novau de Noirmoutier). D'autres blocs se sont effondrés et ont été occupés par la mer : le colmatage récent y a reconquis le Marais de Bouin et celui de Mont.

Mais le Haut-Bocage est resté à l'écart de ces influences : dégagée avant l'éocène par un travail de réadaptation à la structure, la ligne de relief correspondant au contact des schistes et de la granulite qui barre vers le Nord-Est l'horizon de la Vendée, a été comme un môle au pied duquel sont venus mourir la vague d'aplanissement éocène aussi bien que les mouvements récents.

Conclusion. -- Ainsi le schéma semble-t-il le même en Bretagne et dans le Massif Vendéen: une vaste surface ćocène, dominée lès cette époque par un relief de type appalachien où la réadaptation à la structure est plus ou moins avancée, a été relevée dans l'ensemble vers l'intérieur, constituant au pied des hauteurs un large glacis plus ou moins disloqué par des accidents d'importance variable (1), qui sont sans doute assez récents (M. Barrois a indiqué un âge post-miocène pour le Sillon de Bretagne).

Comme nous nous proposons de le montrer bientôt de facon plus complète, surface éocène et mouvements récents jouent dönc dans tout le Sud du Massif Armoricain un rôle beaucoup plus grand qu'on ne l'admettait jusqu'ici et permettenl d'expliquer bien des aspects morphologiques.

11) M. Mriox a invoque lo colmataye de " vastes curettes à fond plat". Mais l'existence de dépôts très dénivelés de chaque côté de grands arei-

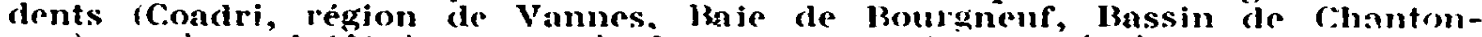

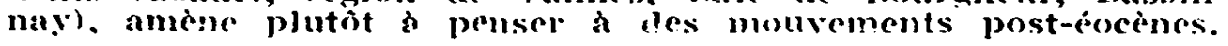

